\title{
Insulin sensitivity: modulation by nutrients and inflammation
}

\author{
Simon Schenk, Maziyar Saberi, and Jerrold M. Olefsky
}

Department of Medicine, Division of Endocrinology and Metabolism, UCSD, La Jolla, California, USA.

\begin{abstract}
Insulin resistance is a major metabolic feature of obesity and is a key factor in the etiology of a number of diseases, including type 2 diabetes. In this review, we discuss potential mechanisms by which brief nutrient excess and obesity lead to insulin resistance and propose that these mechanisms of action are different but interrelated. We discuss how pathways that "sense" nutrients within skeletal muscle are readily able to regulate insulin action. We then discuss how obesity leads to insulin resistance via a complex interplay among systemic fatty acid excess, microhypoxia in adipose tissue, ER stress, and inflammation. In particular, we focus on the hypothesis that the macrophage is an important cell type in the propagation of inflammation and induction of insulin resistance in obesity. Overall, we provide our integrative perspective regarding how nutrients and obesity interact to regulate insulin sensitivity.
\end{abstract}

Obesity is a well-described epidemic in Westernized cultures. In the United States alone, it is estimated that approximately $66 \%$ of all adults are overweight and approximately $32 \%$ are obese (1). With obesity comes a variety of adverse health outcomes, such as high blood pressure, insulin resistance, and type 2 diabetes $(2,3)$. Insulin resistance is defined as an inadequate response by insulin target tissues, such as skeletal muscle, liver, and adipose tissue, to the physiologic effects of circulating insulin. The hallmarks of impaired insulin sensitivity in these three tissues are decreased insulin-stimulated glucose uptake into skeletal muscle, impaired insulin-mediated inhibition of hepatic glucose production in liver, and a reduced ability of insulin to inhibit lipolysis in adipose tissue. In fact, insulin resistance is a major predictor for the development of various metabolic sequelae, including type 2 diabetes (4), and is a defining feature of syndrome $\mathrm{X}$, which is also known as the metabolic syndrome (3). This syndrome encompasses a constellation of conditions, including insulin resistance, dyslipidemia, hypertension, and obesity, and is often accompanied by hyperinsulinemia, sleep apnea, and other disorders (3).

In type 2 diabetes, it has been widely established that insulin resistance precedes the development of overt hyperglycemia (5). The causes of insulin resistance can be genetic and/or acquired. Genetic causes or predispositions toward insulin resistance in prediabetic populations are poorly understood from a mechanistic point of view, although lean, insulin-resistant, prediabetic individuals (e.g., nondiabetic offspring of 2 diabetic parents) can display defects in oxidative metabolism (6). In addition, inherited defects in the basic insulin signaling cascade have been proposed (7). Nonetheless, it is likely that any genetic component must interact with environmental factors in order for insulin resistance to develop into a pathophysiologically meaningful abnormality. In Western cultures, the most common acquired factors causing insulin resistance are obe-

Nonstandard abbreviations used: ATM, adipose tissue macrophage; $\mathrm{CPKC}$, conventional PKC; CPT-I, carnitine palmitoyltransferase I; DAG, diacylglycerol; DGAT, DAG acyltransferase; GPAT, glycerol-3-phosphate acyltransferase; HFD, high-fat diet; IKK, IאB kinase; IMTG, intramuscular TG; LPA, lysophosphatidic acid; mTOR, mammalian target of rapamycin; nPKC, novel PKC; PA, phosphatidic acid; SIRT1, sirtuin 1; TG, triglyceride; UPR, unfolded protein response.

Conflict of interest: J.M. Olefsky serves as a consultant for Pfizer Inc. and Metabolex Inc.

Citation for this article: J. Clin. Invest. 118:2992-3002 (2008). doi:10.1172/JCI34260. sity, sedentary lifestyle, and aging, all of which are interrelated (2, $3,8)$. In the presence of a robust compensatory insulin secretory response to insulin resistance, glucose levels can remain relatively normal. However, when insulin-producing pancreatic $\beta$ cells can no longer compensate for the decreased tissue insulin sensitivity, glucose homeostasis deteriorates and impaired glucose tolerance and eventually frank type 2 diabetes develop (5).

In this review, we provide our personal perspective on the evolving evidence concerning environmental inputs, particularly nutrient availability and pathways that "sense" nutrients, and their interaction with insulin signaling. We then discuss how nutrient availability and obesity interact to modulate inflammatory pathways within liver and adipose tissue. We place particular emphasis on the role of macrophages in this inflammatory process and how this role subsequently affects insulin sensitivity. It is important to note that many other factors, such as the CNS and genetics, are important effectors of obesity, insulin sensitivity, and type 2 diabetes, and readers are referred to additional reviews on these topics (9-11).

\section{Cellular insulin action}

In skeletal muscle and adipose tissue, an increase in insulin promotes glucose uptake by activating a complex cascade of signaling events, which have been reviewed in detail elsewhere $(12,13)$. In brief, binding of insulin to the insulin receptor leads to downstream tyrosine phosphorylation of protein substrates that then engage and activate PI3K. This leads to downstream signaling through PKB/Akt and PKC- $\lambda / \zeta$, which results in GLUT4 translocation from its intracellular pool to the plasma membrane and glucose transport into the cell $(12,13)$. In adipose tissue, insulin is also antilipolytic, whereby it inhibits the release of fatty acids from adipocytes by decreasing the activity of hormone-sensitive lipase and adipose triglyceride lipase (ATGL) (14). In the liver, insulin decreases the release of glucose from the liver by inhibiting hepatic glycogenolysis (glycogen breakdown to glucose) and the expression of key gluconeogenic enzymes (15).

Negative regulation of insulin signaling: links with proinflammatory pathways. A major mechanism by which insulin signaling can be negatively regulated is via phosphorylation of certain serine residues on IRS-1, including Ser312 (Ser307 in the rodent IRS-1 protein), Ser636 (Ser632 in the rodent IRS-1 protein), and Ser1101 (reviewed in ref. 16). This was first revealed in studies investigating 
how the proinflammatory cytokine TNF- $\alpha$ impairs insulin action $(17,18)$. Specifically, phosphorylation of these serine residues impedes the normal association of IRS- 1 with the insulin receptor, thereby impairing downstream propagation of insulin signaling $(16,19)$. Subsequently, a wide variety of factors, including nutrients such as fatty acids and amino acids, have been found to induce insulin resistance at least in part through inhibitory IRS-1 serine phosphorylation (16). It should be noted, however, that phosphorylation of serine residues on IRS-1 is not always inhibitory but rather can positively activate insulin signaling (16). While the precise mechanisms that lead to inhibitory IRS-1 serine phosphorylation are still being elucidated, it is apparent that several serine kinases, including IKB kinase (IKK), JNK, mammalian target of rapamycin (mTOR), and PKC- $\theta$, are involved (16). Interestingly, these serine kinases, in particular IKK and JNK, are integral components of two major proinflammatory pathways (20) and thus provide a potential link between inflammation and insulin resistance. Along these lines, numerous insulin-resistant states (e.g., obesity, type 2 diabetes, and lipid/heparin infusion) are associated with increased activation of JNK $(18,21)$ and/or IKK $(21-25)$ in parallel with inhibitory serine phosphorylation of IRS-1 $(18,21)$. In contrast, inhibition or inactivation of $\operatorname{JNK}(18,26)$ or $\operatorname{IKK}(22$, $24,25,27)$ improves insulin sensitivity in various models of insulin resistance in association with reduced inhibitory serine phosphorylation of IRS-1 (18). Overall, this observation that insulin signaling can be regulated by serine kinases such as JNK and IKK is significant because it provides a potential link between obesity (and excessive fatty acid flux), inflammation, and negative regulation of insulin action.

\section{Nutrient availability and acute modulation of tissue insulin sensitivity}

Caloric overfeeding in rodents that results in a positive energy balance rapidly induces skeletal muscle and liver insulin resistance (28), while calorie restriction in humans and rodents enhances skeletal muscle $(29,30)$ and liver (29) insulin sensitivity. Studies in humans have shown that much of the improvement in insulin sensitivity during weight loss occurs during the first week of dietary restriction and before substantial weight loss occurs (29, 31). Thus, significant changes in insulin action can occur with small perturbations in energy balance, without substantial weight change, and within a relatively short time frame. The fact that insulin sensitivity can be modulated so quickly raises some important conceptual questions, such as, why and how are these tissues so sensitive to nutritional cues? In some ways, this is reminiscent of the effects of gastric bypass surgery to rapidly alleviate insulin resistance and diabetes well before significant weight loss ensues (32). How bypassing the foregut leads to these rapid insulin-sensitizing effects is currently unknown, but perhaps the mechanisms parallel those seen with acute changes in nutrient balance.

Molecular nutrient sensors and insulin action. The development of feed-forward and feedback pathways ensures that during caloric deprivation, the integrated physiologic uptake and storage of nutrients is optimized, while under conditions of overnutrition, the cell is able to limit nutrient uptake. This also implies that there are pathways intrinsic to the cell that can acutely detect or "sense" changes in energy balance or nutritional availability. Indeed, there are a number of pathways that respond to perturbations in caloric load, including the regulatory subunits of PI3K (i.e., p85, p55, p50) $(33,34)$, the protein deacetylase sir- tuin 1 (SIRT1) $(35,36)$, and the serine/threonine protein kinase mTOR (37-39). These pathways are of interest because they are also known to modulate insulin action. The regulatory subunits of PI3K exist either as monomers or as heterodimers bound to the p110 catalytic subunit of PI3K $(40,41)$, although this has recently been questioned (42). Nevertheless, it is hypothesized that during insulin stimulation, when p85-p110, p55-p110 or p50-p110 heterodimers bind to IRS-1, normal insulin signaling occurs, but when monomeric regulatory subunits bind to IRS-1, insulin signaling is competitively inhibited $(40,41)$. Thus, excess regulatory subunits of PI3K can cause decreased insulin sensitivity and are indeed elevated in skeletal muscle from insulin-resistant subjects (21). Importantly, clinical research studies have shown a significant induction of p85 in skeletal muscle after as little as three days of positive energy balance (34). Alternatively, caloric restriction in rodents enhances glucose transport into skeletal muscle in parallel with decreased abundance of p55 and p50 (33). The mTOR/ p70S6 kinase pathway is a putative nutrient-sensing pathway, and overactivation of this pathway in cell-based systems, rodents, and humans is also associated with insulin resistance (37-39). In contrast to these negative regulators of insulin action, SIRT1 appears to be a positive regulator of insulin sensitivity $(43,44)$. Studies in humans have demonstrated increased SIRT1 expression during caloric restriction (36), while caloric overload (via high-fat diet [HFD] feeding) in mice decreases SIRT1 in conjunction with a decrease in insulin sensitivity (35).

\section{Chronic modulation of insulin sensitivity during obesity}

Obesity and the associated increase in body fat mass are the consequences of chronic, long-term (months to years) positive energy balance. As such, obesity differs from "acute" positive energy balance, and the mechanisms of insulin resistance underlying these two conditions are also likely to differ. Insulin resistance in obesity is a reflection of this long-term nutrient excess and is manifested through complex, heterogeneous mechanisms that can involve increased fatty acid flux, nutrient overload, microhypoxia in adipose tissue, ER stress, secretion of adipocyte-derived cytokines, chronic tissue inflammation, genetic predisposition, and CNS inputs. Thus, there is no single cause or set of causes of insulin resistance common to all obese patients. Rather, it is likely that the exact mechanisms of insulin resistance differ across different individual patients and populations. A discussion of all of these factors is beyond the constraints of this Perspective, and below we focus largely on the interactive role of nutrients (particularly fatty acids) and inflammation on the pathogenesis of insulin resistance.

Fatty acid flux and insulin sensitivity. Obese individuals are characterized by a greater rate of breakdown and uptake (i.e., flux) of fatty acids as compared with lean individuals (45), and it is likely that this higher flux is an important mediator of insulin resistance. It is well known that increasing the flux of fatty acids in lean individuals to rates similar to or greater than those seen in obese individuals (typically via a lipid-plus-heparin infusion) induces insulin resistance $(46,47)$. It should be noted that a lipid infusion does not recapitulate the obese state; however, it does provide a unique means of mimicking the high fatty acid flux found in obesity without the presence of the other potentially confounding variables associated with increased adiposity. Further evidence linking fatty acids to insulin sensitivity comes from clinical studies showing that pharmacological inhibition of lipolysis decreases fatty acid levels and rapidly enhances insulin sen- 


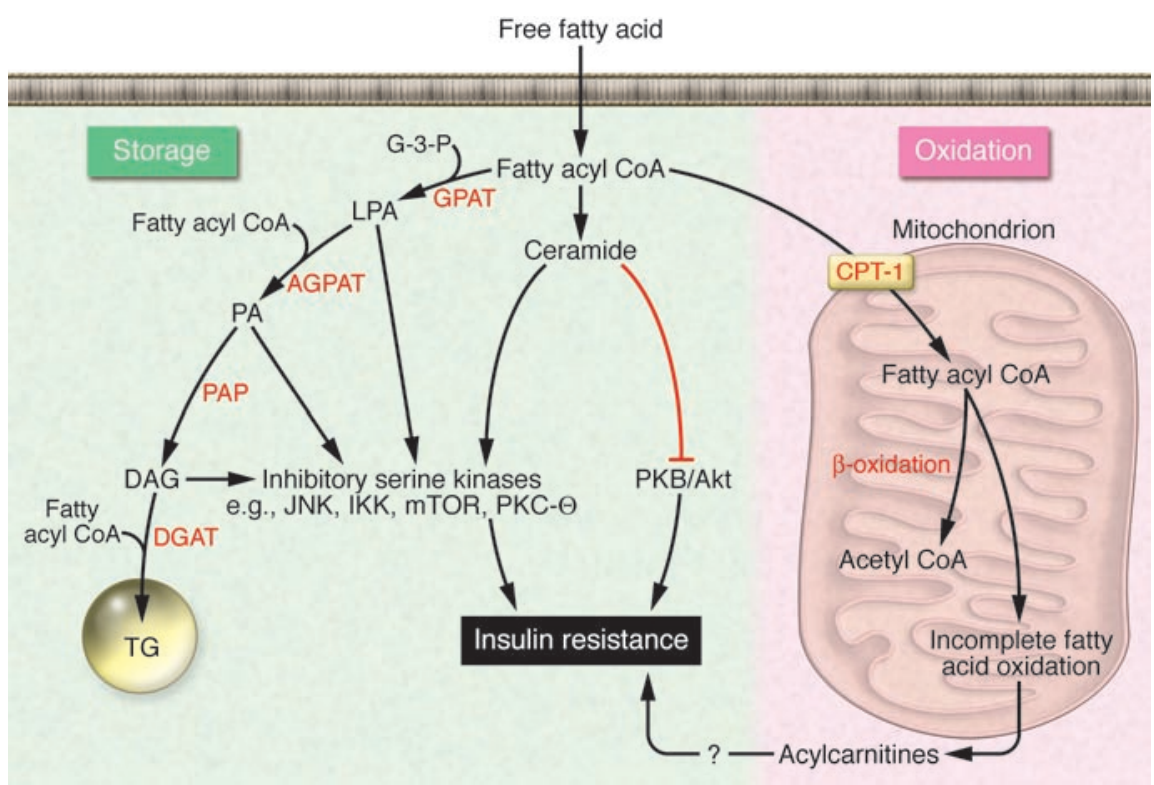

Figure 1

Fatty acid metabolism and insulin action in skeletal muscle or liver. Obesity results in an increased flux of free fatty acids into the circulation and uptake by the myocyte or hepatocyte. Activated fatty acids (i.e., fatty acyl-CoAs) are "metabolized" primarily via one of two pathways, oxidation or storage. When fatty acid flux exceeds the ability of these pathways to dispose of fatty acyl-CoAs, intermediaries of fatty acid metabolism (e.g., DAG, PA, LPA, ceramide) accumulate. In turn, these fatty acid intermediates can activate a number of different serine kinases that can negatively regulate insulin action. Ceramide can also impair insulin action through interactions with PKB/Akt. An inability to completely oxidize fatty acids through $\beta$-oxidation, which leads to an accumulation of acylcarnitines, has also been hypothesized to cause insulin resistance, although the precise mechanisms leading to insulin resistance are, to date, unknown. AGPAT, acylglycerol-3-phosphate acyltransferase; PAP, PA phosphohydrolase. those with 15-17 carbons (e.g., palmitate) is high (52). It has been hypothesized that when the efficiency of skeletal muscle to dispose of fatty acids via oxidation or storage is maximized, these various fatty acid "intermediates" (i.e., DAG, ceramide, etc.) accumulate in skeletal muscle and liver and negatively regulate insulin action (6, $47,53)$. As discussed below and shown in Figure 1, there are several potential mechanisms by which fatty acid metabolism can regulate insulin action.

IMTG, fatty acid intermediates, and insulin action. The concentration of IMTG, fatty acyl-CoAs, DAG, and/or ceramide are elevated in skeletal muscle from obese humans, genetically obese rats, and HFD-fed, obese mice in association with insulin resistance (53-57). This accumulation appears to be related to the increased fatty acid flux seen in obesity. Accordingly, increasing fatty acid flux via a lipid-plus-heparin infusion in humans $(23,46)$ or rodents $(58)$ results in a corresponding increase in the concentration of IMTG and/or fatty acid intermediates. In contrast, decreasing fatty acid flux in humans via pharmacological inhibition of lipolysis (49) or by discontinuing a lipid-plus-heparin infusion in rats (58) decreases IMTG concentration and/or the accumulation of fatty acid intermediates. Interestingly, a number of these fatty acid intermediates activate the aforementioned proinflammatory and nutrient-sensing sitivity in diabetic and nondiabetic, lean and obese subjects (48, 49). Weight loss also decreases fatty acid flux (50) and improves insulin sensitivity $(29,31)$, but the quantitative contribution of this decrease in fatty acid flux to the improvement in insulin sensitivity has not been determined.

Fatty acid metabolism and insulin sensitivity. When fatty acids are taken up by muscle (or liver), they undergo two primary routes of disposal (Figure 1). They can undergo $\beta$-oxidation in the mitochondria, or they can be stored as triglycerides (TGs). It should be noted that in the liver, there are two TG pools, the intracellular hepatocyte TG pool and the VLDL TG pool, which is subsequently secreted into the systemic circulation (51). The rate-limiting step for $\beta$-oxidation of long-chain fatty acids is their transport into the mitochondria via carnitine palmitoyltransferase-I (CPT-I). The synthesis of TG (in skeletal muscle this is often referred to as intramuscular TG [IMTG]) from fatty acids entails the sequential addition of activated fatty acyl-CoA moieties to a glycerol backbone (51). The first committed step in TG synthesis is catalyzed by glycerol-3-phosphate acyltransferase (GPAT), which results in the synthesis of lysophosphatidic acid (LPA). In subsequent steps, LPA is converted to phosphatidic acid (PA), and PA is converted to diacylglycerol (DAG). The final step in TG synthesis is the addition of a fatty acyl-CoA to DAG by DAG acyltransferase (DGAT). Another important product of fatty acid metabolism that is relevant to insulin action is ceramide, which is synthesized when the availability of saturated fatty acids, particularly pathways. LPA, PA, DAG, and ceramide can activate the mTOR/ p70S6K, JNK, IKK, and/or the novel PKC/conventional PKC (nPKC/cPKC; e.g., PKC- $\theta$ ) pathways (59-62). Ceramide can also (63). Indeed, lipid-induced insulin resistance in rats is prevented by pharmacological inhibition of ceramide production (57). Similarly, pharmacological inhibition and/or genetic ablation in cellbased, mouse, or human models of the mTOR/p70S6K (37-39), IKK/NF-кB $(24,25,27,64)$, JNK $(18,26)$, or nPKC/cPKC (65) pathways prevents lipid-, obesity-, and nutrient overload-induced insulin resistance.

A cause-effect relationship between IMTG levels and insulin sensitivity has recently been questioned $(47,53,55)$. Perhaps the most widely recognized discrepancy between IMTG levels and insulin sensitivity is the "athlete's paradox," in which endurance-trained athletes have similar (or even higher) IMTG levels than obese and type 2 diabetic individuals yet are highly insulin sensitive (55). One hypothesis to explain this paradox is that increasing IMTG synthesis decreases the accumulation of the aforementioned fatty acid intermediates, thereby limiting their negative downstream effects on insulin action. To this end, muscle-specific overexpression of Dgat1 as well as an acute bout of exercise (which increases mitochondrial GPAT and DGAT1 abundance) prevents HFD- and lipid-induced impairments in insulin action in conjunction with increased IMTG synthesis and decreased DAG and ceramide accumulation as well as reduced activation of $\mathrm{nPKC} / \mathrm{cPKC}, \mathrm{JNK}$, and/or attenuate insulin signaling through its ability to inhibit Akt/PKB 
IKK/NF-кB $(47,53)$. These results are comparable to findings in which increasing the capacity of adipose tissue (66) or the liver (67) to incorporate fatty acids into TGs does not cause insulin resistance and can actually prevent HFD-induced insulin resistance (66).

Fatty acid oxidation, mitochondrial dysfunction, and insulin resistance. In recent years, the capacity of muscle to oxidize fatty acids has received much attention as a key modulator of insulin action. Skeletal muscle from various insulin-resistant subjects displays reduced CPT-I activity and/or fatty acid oxidative capacity (reviewed in ref. 68 ) as well as decreased expression of genes involved in modulating oxidative metabolism and mitochondrial biogenesis $(69,70)$. The mitochondrial compartment in obese and insulin-resistant individuals has also been hypothesized to be dysfunctional, and this mitochondrial dysfunction could directly contribute to the pathogenesis of insulin resistance (reviewed in ref. 6). From the perspective of the whole muscle tissue bed, mitochondrial dysfunction could be due to a decreased number of normally functioning mitochondria or a defect in individual mitochondria per se.

Mechanistically, a reduced ability of skeletal muscle to oxidize fatty acids when fatty acid flux is high (such as in obesity) would lead to an accumulation of fatty acid intermediates; activation of the JNK, IKK, and/or nPKC/cPKC pathways; and impaired insulin action. Increasing the ability of skeletal muscle to oxidize fatty acids could reduce the accumulation of fatty acid intermediates and therefore improve insulin sensitivity. While this may be true in certain conditions (71), it is notable that endurance-trained athletes, who have a very high capacity to oxidize fatty acids, exhibit a similar fatty acid-induced decrease in insulin action as compared to lean controls during a lipid-heparin infusion (72). In addition, although numerous studies (reviewed in refs. 6,68 ) have demonstrated reduced fatty acid oxidative capacity and impaired mitochondrial function in skeletal muscle from obese and type 2 diabetic individuals, a number of in vitro and in vivo reports have not supported these findings. Thus, clinical studies have demonstrated that obese, insulin-resistant, and type 2 diabetic individuals do not exhibit a reduced capacity to oxidize fatty acids $(73,74)$ and have normal, or even increased, mitochondrial function, as compared with lean controls $(56,75)$. It is possible that some of these discrepancies and results may be related to differences in the populations studied. For example, a recent report showed that nondiabetic Asian Indians were highly insulin resistant when compared with appropriately matched northern Europeans, even though skeletal muscle from these subjects demonstrated a greater capacity for oxidative phosphorylation (76). In fact, this elevated capacity for oxidative phosphorylation was maintained in skeletal muscle from diabetic Asian Indians, yet they were hyperglycemic and displayed severe insulin resistance (76). Studies in rodents have also failed to support a cause-and-effect relationship between mitochondrial number/function and insulin sensitivity. Reducing mitochondrial number and the expression of oxidative and mitochondrial genes in skeletal muscle via $\mathrm{KO}$ of peroxisome proliferator coactivator $\gamma-1$ (PGC-1), which is a transcriptional regulator of mitochondrial biogenesis, in mice does not cause skeletal muscle insulin resistance (77). Similarly, induction of mitochondrial dysfunction in skeletal muscle does not impair skeletal muscle insulin sensitivity (78), nor does mitochondrial dysfunction appear to precede the onset of HFD-induced insulin resistance in mice (79). Thus, although mitochondrial dysfunction has recently gained much attention as a potential hypothesis explaining insulin resistance in obesity and diabetes, further research is required. Along these lines, even in studies where a reduced in vivo capacity to oxidize fatty acids has been noted, it still remains to be determined whether this mitochondrial dysfunction is a direct cause of insulin resistance in obesity or is a secondary consequence of obesity/insulin resistance and/or reduced physical activity.

Recently, an alternate hypothesis connecting fatty acid oxidation to insulin resistance has been proposed (80). According to this alternate view, rather than fatty acid oxidation being reduced in insulin-resistant states, insulin resistance in skeletal muscle is accompanied by excessive rates of $\beta$-oxidation and mitochondrial overload. Specifically, when a fatty acid is metabolized via $\beta$-oxidation, each cycle produces an acetyl-CoA molecule and shortens the fatty acid chain by 2 carbons. However, when $\beta$-oxidation exceeds the ability of the mitochondria to utilize acetyl-CoA in the TCA cycle, there is a resultant buildup of incompletely oxidized, shorterchain fatty acid products (i.e., acylcarnitines), which, via a yet-tobe-identified mechanism, can impair skeletal muscle insulin sensitivity (80). Indeed, skeletal muscle from insulin-resistant HFD-fed mice and Zucker diabetic fatty (ZDF) rats exhibits an increased abundance of partially oxidized acylcarnitines (80). Furthermore, mice with $\mathrm{KO}$ of malonyl-CoA decarboxylase, which reduces the rate of entry of fatty acyl-CoAs into the mitochondria, have lower skeletal muscle levels of acylcarnitines on a HFD, in parallel with improved whole-body insulin sensitivity (80).

An additional factor contributing to skeletal muscle insulin resistance may relate to differences in muscle fiber type. For example, aerobic fitness training leads to increased type I, oxidative muscle-fiber capacity in association with improved insulin sensitivity (81). On the other hand, recent studies indicate that an increase in type IIb glycolytic muscle fibers, which are usually enhanced with resistance training, can also improve insulin sensitivity (82). Thus, the contribution of specific muscle fiber types to systemic insulin sensitivity in humans remains to be fully defined.

\section{Inflammation: a link between obesity and insulin resistance}

Many lines of evidence have shown that chronic activation of proinflammatory pathways within insulin target cells can lead to obesity-related insulin resistance. Consistent with this, elevated levels of the proinflammatory cytokines TNF- $\alpha$, IL-6, and C-reactive protein (CRP) have been shown in individuals with insulin resistance and diabetes (for review, see refs. 83-85). Furthermore, TNF- $\alpha$ levels are elevated in adipose tissue and blood from obese rodents, and neutralization of TNF- $\alpha$ improves insulin sensitivity in these animals (86). As discussed below, fatty acids, microhypoxia in adipose tissue, ER stress, and certain cytokines can all initiate proinflammatory responses by activating the JNK/activator protein 1 (AP1) and IKK/NF-KB signaling pathways. JNK $(18,21)$ and $\operatorname{IKK}(22,25)$ signaling are upregulated and activated in insulin-resistant human and rodent skeletal muscle. This is important, as these serine kinases phosphorylate the transcription factor targets AP1 (c-Jun/Fos) and NF- $\mathrm{KB}$, which then transcriptionally activate an overlapping set of inflammatory pathway genes (20), ultimately leading to decreased insulin sensitivity. Accordingly, $\mathrm{KO}$ or inhibition of JNK1 or IKK $\beta$ prevents insulin resistance in cell and mouse models $(18,22,24,25,87)$ as well as human models (27) of insulin resistance.

An important initiator of this inflammatory response is adipose tissue, which is not only a storage depot for excess calories but also actively secretes fatty acids and a variety of polypeptides. These 


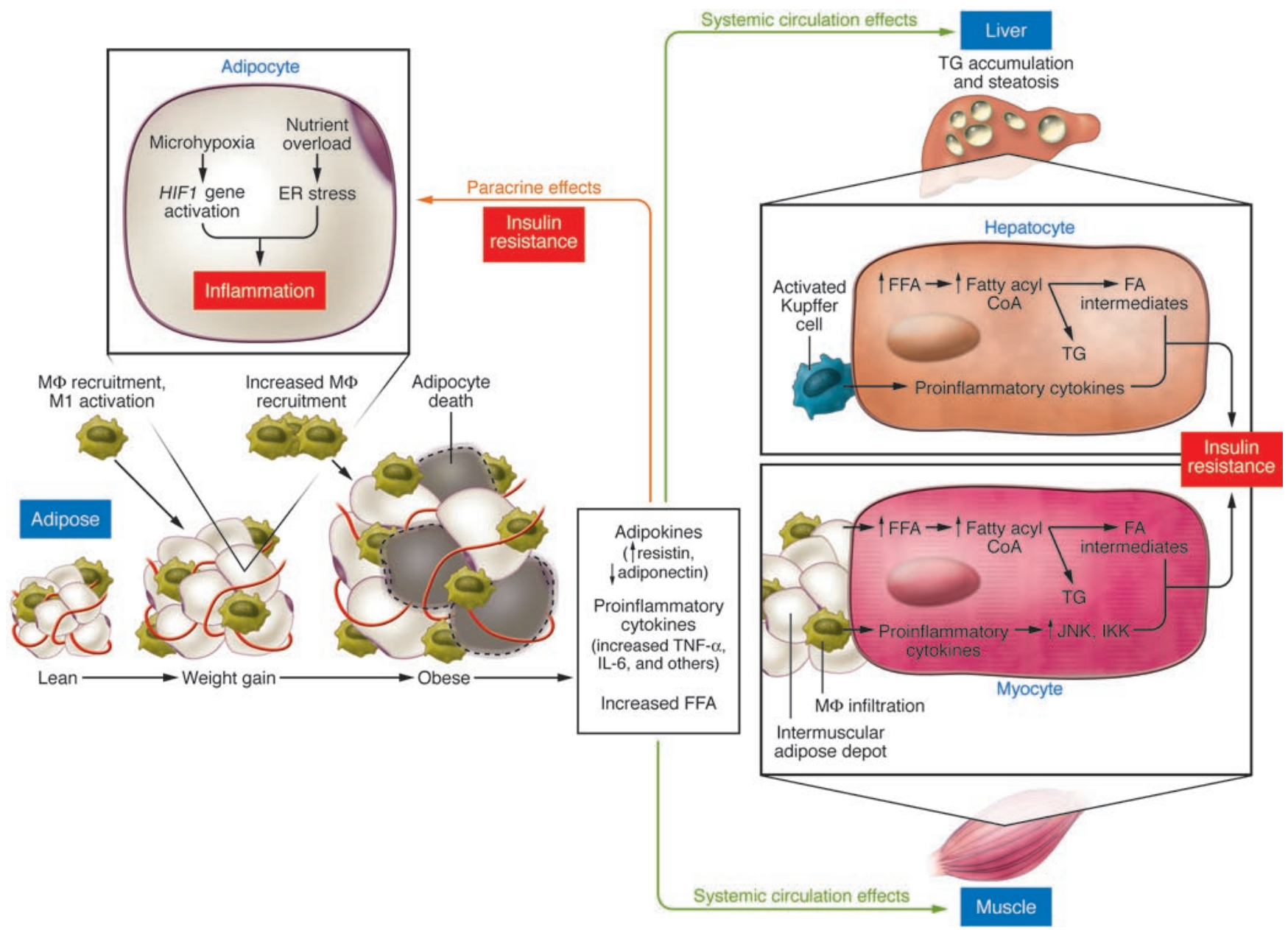

Figure 2

Obesity, tissue inflammation, and insulin resistance. Nutrient excess, weight gain, and ensuing obesity result in expansion of adipose tissue mass and adipocyte size. With this expansion, total free fatty acid release into the circulation is increased and oxygen delivery to the adipocyte is decreased. The combination of microhypoxia and nutrient excess leads to induction of HIF-1 and the downstream target genes as well as ER stress within the adipocyte. This can lead to the eventual death of the adipocyte as well as a characteristic inflammatory response. The inflammatory response includes increased production and release of proinflammatory cytokines/chemokines and the recruitment of bone marrow-derived macrophages $(\mathrm{M} \phi)$. These macrophages are of the $\mathrm{M} 1$ activation/polarization state and are highly inflammatory in nature. Once recruited, these macrophages release proinflammatory cytokines, which work in a paracrine manner to activate the intracellular proinflammatory pathways (e.g., JNK and IKK) in neighboring cells and possibly through endocrine mechanisms in distal tissues. In a feed-forward cycle, activation of macrophages promotes the recruitment and infiltration of additional macrophages into adipose tissue. This results in cell autonomous insulin resistance in adipocytes and liver, exacerbation of the inflammatory state, and systemic insulin resistance. With obesity, there is also increased fat accumulation within skeletal muscle, and these intermuscular fat depots becomes infiltrated with proinflammatory macrophages, which may cause paracrine-like insulin resistance in skeletal muscle. In parallel with these inflammation-related changes, alterations in fatty acid metabolism can lead to the accumulation of fatty acid intermediates with the liver and skeletal muscle, which can cause insulin resistance via mechanisms outlined in Figure 1. In addition, fatty acids can serve as ligands to broadly activate inflammatory pathways in Kupffer cells and ATMs (e.g., via TLR2/TLR4 signaling pathways).

peptides include hormones, cytokines, and chemokines that can function in both an endocrine or paracrine fashion. The adipose tissue itself consists of a variety of cell types including adipocytes, immune cells (macrophages and lymphocytes), preadipocytes, and endothelial cells, etc. Several cytokines and chemokines, such as CCL2, IL-6, IL-1 $\beta$, macrophage migration inhibitory factor (MIF), and TNF- $\alpha$, can be released by adipocytes and macrophages (83-85). Adipocytes are the unique source of secreted adipokines such as leptin and adiponectin, which can promote insulin sensitivity, as well as resistin and retinol-binding protein 4 (RBP4), which can impair insulin sensitivity (83-85). Thus, the mixture of adipokines secreted by adipose tissue in a given pathophysiologic state can have important effects on systemic insulin sensitivity. However, a detailed description of these various adipokines and their actions is beyond the constraints of this review, and the reader is directed to other recent reviews on this subject (83-85). The events that potentially link obesity to the initiation and propagation of inflammation and that subsequently link inflammation to the induction of tissue and systemic insulin resistance are detailed below and are illustrated in Figure 2. 


\section{Pushing the button: initiating inflammation}

Adipocyte hypertrophy, adipocyte cell death, and macrophage recruitment and inflammation. As the adipose tissue bed expands during the progression to obesity, the enlarging adipocytes become relatively hypoperfused, which creates regional areas of microhypoxia and in turn leads to increased expression of the HIF-1 gene program (88-90). Hypoxic adipose tissue displays enhanced activation of the JNK1 and IKK/NF-KB pathways as well as increased expression of genes involved in inflammation and ER stress (88-90). Activation of these pathways leads to chemokine release and subsequent recruitment of macrophages into the adipose tissue, where they form ring-like structures around large, dying adipocytes (90-92). The primary purpose of these recruited macrophages is the removal of cell debris and tissue remodeling. In addition, these macrophages release proinflammatory cytokines, which can induce insulin resistance in neighboring adipocytes via paracrine effects. It is also possible that this adipose tissue microhypoxia exacerbates the proinflammatory nature of the newly recruited macrophages, resulting in further activation of hypoxia-sensitive pathways and amplification of the inflammatory state. These events appear to be more pronounced in visceral adipose tissue compared with subcutaneous fat depots, as expansion of visceral fat has a uniquely adverse effect on insulin sensitivity (93).

ER stress and inflammation. The ER is a specialized organelle that functions as a cholesterol and nutrient sensor in addition to its well-established role in de novo protein synthesis and protein folding (94-96). Under normal conditions, proteins are folded and assembled by chaperones in the ER (94-96). However, under conditions of hypoxia or the inundation of the cell and ER with nutrients, such as fatty acids, the unfolded protein response (UPR) cascade can be activated (94-96). The UPR can be triggered by changes in redox state, alteration in $\mathrm{Ca}^{2+}$ levels sensed by the $\mathrm{ER}$, or accumulation of unfolded or misfolded proteins in the ER lumen (94-96). Activation of the UPR leads to transcriptional induction of a number of genes involved in the assembly, folding, modification, and degradation of proteins to alleviate ER stress (94-96). The reader is referred to several excellent recent reviews for complete details on the ER stress response (94-96). Ozcan et al. (97) have shown that activation of the UPR increases JNK activity and Ser307 phosphorylation of IRS-1, in association with impaired insulin action. The UPR also leads to increased IKK $\beta$ and proinflammatory cytokine expression (e.g., Il6, Tnfa, and Ccl2) (94, $96,98)$. In further support of a role for ER stress in the induction of insulin resistance, $\mathrm{KO}$ of X-box protein 1 (Xbp1), a gene encoding a transcription factor that mediates expression of ER chaperones, leads to increased ER stress, JNK activation, IRS-1 Ser307 phosphorylation, hyperinsulinemia, and glucose intolerance in HFD-fed mice (97). Treatment of HFD-fed mice with orally active chemical chaperones reverses these effects on ER stress and JNK and improves tissue insulin sensitivity (99).

While ER stress can link established obesity to insulin resistance, whether the acute decrease in insulin sensitivity caused by overfeeding is due to ER stress is unclear. A recent study in transgenic mice that express an ER stress-activated GFP reporter did not detect an ER stress response in the adipose tissue after 12 weeks of HFD feeding but did detect ER stress in the liver as early as 4 weeks (100). In other studies, ER stress was detected in adipose tissue after 12-14 weeks of HFD feeding (99). Overall, these data suggest that ER stress-induced inflammation and insulin resistance are caused by chronic nutrient excess (i.e., obesity), rather than acute nutrient overload. Interestingly, although substantial evidence demonstrates that chronic nutrient overload can trigger ER stress in adipose tissue and liver, to date, no studies have demonstrated obesity-induced ER stress in skeletal muscle $(97,100)$.

\section{Propagating the inflammatory signal: the role of the macrophage}

In 2003, the novel discovery that adipose tissue from obese mice and humans is infiltrated with macrophages provided a major mechanistic advance into understanding how obesity propagates inflammation $(101,102)$. In these studies, adipose tissue was shown to contain bone marrow-derived macrophages, and the content of these macrophages tracked with the degree of obesity $(101,102)$. In fact, more than $40 \%$ of the total adipose tissue cell content from obese rodents and humans was comprised of macrophages compared with approximately $10 \%$ in lean counterparts (101). In addition, macrophages were responsible for the majority of Tnfa expression in adipose tissue and contributed significantly to $I l 6$ and $I k b k b$ expression $(101,102)$. Increased infiltration of obese adipose tissue with macrophages in mice and humans has subsequently been verified in many studies (87, 90-92, 103-106). Such studies have focused on how and why macrophages are recruited to the adipose tissue and whether macrophage-induced inflammation can mediate insulin resistance.

Macrophages link inflammation to insulin resistance. The JNK and IKK/NF-кB pathways represent important modulators of inflammatory gene expression in many cell types, including skeletal muscle, liver, and adipose tissue. In macrophages, depletion of JNK1 and IKK $\beta$ inhibits proinflammatory gene expression and cytokine release in response to fatty acids or LPS $(87,107)$. To better understand the role of macrophages in the propagation of inflammation and the induction of insulin resistance, myeloid cell (i.e., macrophages, lymphocytes, and neutrophils) IKK $\beta$ - or JNK1-KO mice were generated using the Cre-LoxP system (24) or bone marrow transplantation techniques (87). In this way, the macrophage inflammatory pathway was disabled while the expression of IKK $\beta$ and JNK1 remained intact in all other tissues, including liver, adipose, and skeletal muscle. The results showed that myeloid cell KO of IKK $\beta$ or JNK1 protected mice from HFD-induced glucose intolerance, hyperinsulinemia, and insulin resistance in skeletal muscle, adipose tissue, and the liver $(24,87)$. In these studies, the mice developed the same degree of obesity as wild-type littermates, demonstrating that without the myeloid cell-driven inflammatory component, obesity itself did not cause insulin resistance. Consistent with these results, mice deficient in myeloid cell TNF- $\alpha$ are protected against obesity-induced insulin resistance (108). In a similar vein, Cbl-b is a ubiquitin ligase that plays an important role as a negative regulator of macrophage activation and infiltration into tissues. Recent studies show that mice with $\mathrm{KO}$ of Cbl-b have increased numbers of adipose tissue macrophages (ATMs) and heightened levels of inflammatory markers in adipose tissue. These mice also exhibit glucose intolerance, hyperinsulinemia, and insulin resistance, which is consistent with a role of macrophages in causing decreased insulin sensitivity (109).

While macrophages are thought to be the most prominent immune cells underlying the proinflammatory tissue response that leads to insulin resistance, it should be noted that the conventional conditional $\mathrm{KO}$ technologies (e.g., lysozyme M Cre or bone marrow transplantation) also result in target gene depletion in other hematopoietic and immune cells, including neutrophils and 
lymphocytes. Since Th1 lymphocytes can induce proinflammatory responses $(110,111)$ and can attract macrophages into sites of inflammation (111), it is possible that lymphocytes also play some role in the process of inflammation and insulin resistance.

Macrophage heterogeneity and polarization. ATMs display heterogeneous inflammatory behavior, such that discrete cellular populations can be identified. In vivo macrophage pulse-labeling studies showed that macrophages recruited to adipose tissue during induction of obesity with HFD feeding are proinflammatory, as indicated by increased expression of cytokines (e.g., TNF- $\alpha$ ) and inflammatory pathway genes (104). Expression of genes important for macrophage activation, adhesion, and migration is also increased in these macrophages (104). In contrast, the macrophages resident in adipose before HFD feeding are antiinflammatory in nature (104).

Macrophage phenotypes exist across an M1 to M2 polarization state in which M1 cells are operationally defined as "classically activated," proinflammatory macrophages, while M2 cells make up the "alternatively activated," antiinflammatory macrophage population (110). M1 macrophages are induced by proinflammatory factors and display increased inflammatory gene expression with enhanced reactivity to fatty acids and LPS (110). In obesity, the major component of the increased ATM content is composed of proinflammatory macrophages that are triply positive for the surface markers F4/80, CD11b, and CD11c (92, $105)$. Accordingly, because of their proinflammatory properties, these macrophages are implicated in the pathogenesis of insulin resistance $(103,105,112,113)$.

M2 macrophages display low levels of inflammatory gene expression, secrete high levels of antiinflammatory factors, such as IL-10 and IL-4, and are poorly activated by fatty acids and LPS (110). Similar to M1 macrophages, they are positive for F4/80 and CD11b, but they are negative for CD11c (105). Importantly, the PPAR $\gamma$ nuclear receptor appears to be required for the maturation of alternatively activated macrophages $(112,113)$. Treatment of macrophages with the insulin-sensitizing drugs thiazolidinediones (TZDs), which are PPAR $\gamma$ ligands, polarizes macrophages toward the M2/antiinflammatory state, thus providing a potential mechanism by which activation of macrophage PPAR $\gamma$ reduces inflammation and enhances insulin sensitivity (106).

Triggering macrophage activation. While it is clear that proinflammatory macrophages are recruited to adipose tissue in obesity, the pathophysiologic factors that activate these cells are also of interest. Clearly, ATMs exist in a lipid-rich environment and fatty acids can broadly activate the macrophage inflammatory program $(105,114-116)$. An important component of these fatty acid-induced activation events are the pattern recognition receptors TLR2 and TLR4 (105, 114-116). Activation of inflammation is prevented in macrophages and adipocytes that are deficient in Tlr2, Tlr4, or Tlr2/Tlr4 (105, 114-116). In line with this, Tlr4-KO mice are protected against lipid-and obesity-induced insulin resistance $(116,117)$. Interestingly, the expression of Tlr2/Tlr4 is increased in macrophages that are triply positive for F4/80, CD11b, and CD11c (105), thus providing an additional mechanism of feed-forward activation of inflammation within macrophages and the surrounding adipocytes. LPS also signals through TLR4 (in concert with the CD14 receptor), and a recent study found that circulating LPS derived from gastrointestinal bacteria could play a role in insulin resistance. These workers found increased circulating levels of gut-derived
LPS in HFD-fed mice and demonstrated that mice with $\mathrm{KO}$ of Cd14, which is a receptor for LPS, were protected from HFDinduced insulin resistance (118). In addition to fatty acids and LPS, cytokine secretion from the expanding adipose tissue, adipose tissue microhypoxia, and/or ER stress likely also activate or exacerbate macrophage inflammatory pathways.

Recruiting macrophages to adipose tissue. The precise mechanisms that regulate the recruitment of new macrophages to adipose tissue are unclear. CCL2 (also known as monocyte chemoattractant protein 1) is a chemokine that promotes the recruitment of monocytes to damaged or inflamed tissues, and CCR2 is the receptor for CCL2. Ccl2 and/or Ccr2 expression is increased in obese adipose tissue (119-122), and overexpression of CCl2 in adipose tissue increases macrophage infiltration in parallel with reduced insulin sensitivity (121). In addition, $\mathrm{KO}$ of $\mathrm{Ccl} 2$ or $\mathrm{Ccr} 2$ or neutralization of CCL2 leads to decreased macrophage recruitment to adipose tissue and protection from obesity-induced insulin resistance $(120,121)$. In contrast, other studies have found no effect of KO of $C c r 2$ or $C c l 2$ on total macrophage recruitment $(119,122,123)$ or insulin sensitivity $(122,123)$. Thus, the contribution of this ligand/receptor system to macrophage recruitment inflammation and insulin resistance remains to be completely defined. A number of additional factors have been identified as possible modulators of macrophage recruitment to adipose tissue. The cytokine osteopontin is a chemoattractant that is secreted by macrophages in order to facilitate immune cell recruitment, adhesion, and migration. Accordingly, the expression of osteopontin is elevated in obese adipose tissue (124) and osteopontin-KO mice have reduced ATM content after HFD feeding and are protected against HFDinduced insulin resistance (124).

Kupffer cells. The bone marrow-derived macrophage cell type in the liver is the Kupffer cell. Similar to the paracrine role of macrophages in obese adipose tissue, activation of proinflammatory pathways in Kupffer cells could be central to hepatic insulin resistance in obesity. In line with this, myeloid cell-specific JNK1-KO mice (which also exhibit KO of JNK1 in Kupffer cells) develop the full degree of hepatic steatosis on an HFD but do not display increased hepatic markers of inflammation and do not develop hepatic insulin resistance (87). Thus, in the absence of an inflammatory response, steatosis and hepatic insulin resistance can be dissociated. This suggests that in obesity, inflammatory pathways are activated in Kupffer cells, which leads to the release of cytokines and subsequent activation of inflammatory pathways in nearby hepatocytes. In turn, this contributes to hepatic insulin resistance. Consistent with this idea, primary genetic activation of inflammatory pathways within hepatocytes via hepatocyte-specific overexpression of IKK $\beta$ in mice causes hepatic insulin resistance (25). On the other hand, inhibition of NF-KB (25) or knockout of IKK $\beta$ in the hepatocyte (24) improves hepatic insulin sensitivity, indicating that when intrahepatocyte inflammatory pathways are blocked, the ability of the paracrine effects of activated Kupffer cells to cause liver insulin resistance is attenuated. Altogether, these results are consistent with a "two-hit" process in which the activated macrophage (in this case, Kupffer cells) initiates the inflammatory process by secreting proinflammatory cytokines, which subsequently stimulate (in a paracrine fashion) inflammatory pathways within neighboring hepatocytes. It is possible that additional factors also contribute to hepatocyte inflammatory pathway activation, such as intracellular accumulation of fatty acid intermediates. 


\section{Table 1}

Characteristic properties of macrophages in adipose tissue in the lean or obese state

\begin{tabular}{lcc} 
Macrophage characteristics & Lean & $\begin{array}{c}\text { Obese } \\
\text { Macrophage infiltration }\end{array}$ \\
$\begin{array}{l}\text { Activation/polarization } \\
\text { M1 }\end{array}$ & Low & High \\
M2 & Low & High \\
F4/80 and CD11b expression & Positive & Unchanged \\
CD11c expression & Negative & Positive \\
TLR2/TLR4 expression & Low & High \\
Inflammatory state & Low & High \\
Cytokine secretion & Antiinflammatory & Proinflammatory \\
& profile & profile \\
\hline
\end{tabular}

\section{Concluding remarks}

In this Perspective, we first discussed possible factors that acutely (i.e., hours to days) modulate insulin sensitivity, independently of significant weight gain or weight loss. This centered primarily on nutrient "sensors" within skeletal muscle and liver and their interactions with the insulin-signaling cascade. These sensors can detect perturbations in nutrient availability and, depending on the nutrient status of the cell, regulate insulin action and nutrient (i.e., glucose) uptake. These processes provide a means of regulating insulin sensitivity that is cell autonomous (i.e., intrinsic to the cell) and independent of extracellular stimuli. Most likely these effects are distinct from the mechanisms of chronic inflammation- and obesity-induced insulin resistance, summarized below.

Potential mechanisms of chronic insulin resistance in obesity are summarized in Figure 2. This long-term regulation of insulin sensitivity is primarily related to expansion of adipose tissue mass, which leads to increased systemic fatty acid flux, microhypoxia in adipose tissue, induction of ER stress, and ultimately, adipose tissue inflammation. In relation to fatty acid flux and metabolism, as summarized in Figure 1 (and integrated into Figure 2), in obesity, the capacity of myocytes and hepatocytes to fully metabolize fatty acids is generally insufficient to match the excessive fatty acid uptake by cells. This can lead to the cellular accumulation of fatty acid intermediates (e.g., DAG, ceramide, acylcarnitines), which ultimately results in activation of a number of serine kinases, including JNK1, IKK $\beta$, and PKC- $\theta$. The activation of these serine kinases is significant because these kinases represent a convergence point central to inflammationinduced insulin resistance.

In parallel with the perturbations in fatty acid metabolism in skeletal muscle and the liver, adipocyte microhypoxia and ER stress precipitate a series of events that result in the recruitment of a specific population of proinflammatory, M1-like macrophages into adipose tissue. The characteristic properties of these macrophages are presented in Table 1. Activation of these macrophages leads to the release of a variety of chemokines (which recruit additional macrophages) and proinflammatory cytokines. In turn, these cytokines initiate a paracrine process that causes activation of proinflammatory pathways within nearby insulin target cells, such as adipocytes and hepatocytes, leading to cell autonomous insulin resistance in these cells. In the adipocyte, this exacerbates lipolysis, while in the hepatocyte, hepatic glucose production is increased. Thus, this cycle provides a mechanism for the inflammation-related component of insulin resistance in liver and adipose tissue.

The mechanisms by which inflammatory processes contribute to skeletal muscle insulin resistance are less clear. One likely contributory factor is that the macrophage-mediated inflammatory process within adipose tissue and liver changes the milieu of secreted circulating adipokines and/or cytokines, which then have endocrine effects to reduce insulin sensitivity in skeletal muscle. The possibility also remains that inflamed adipose and/ or liver tissue releases heretofore-unidentified metabolites or novel peptides that have effects on insulin sensitivity in skeletal muscle. These possible endocrine effects could be exacerbated by the fact that inflammatory cytokines, such as TNF- $\alpha$, stimulate adipocyte lipolysis, thereby enhancing fatty acid flux (14). The skeletal muscle is also interspersed with adipose tissue, and this depot is increased in obesity and is a strong predictor of insulin resistance (125). Moreover, this intermuscular adipose depot is infiltrated with approximately 3 times more macrophages in skeletal muscle from obese compared with lean mice (101). Similar to the paracrine effects of macrophages in adipose tissue and liver, these macrophages could produce localized proinflammatory cytokines that affect skeletal muscle insulin sensitivity. Altogether, these possibilities suggest that the expanding adipose tissue mass is the primary cause of systemic insulin resistance in obesity and that the disordered homeostasis, inflammation, and insulin resistance that occur in adipose tissue communicate with skeletal muscle, causing skeletal muscle insulin resistance.

From a more general perspective, chronic tissue inflammatory responses (and macrophage recruitment) serve a normal physiologic purpose, such as host defense, tissue repair, or adaptive restoration of tissue homeostasis in response to cellular stress. Often, however, tissue inflammation becomes chronic or remains unresolved and progresses to a pathophysiologic condition. For example, in other disease states, such as cancer and Alzheimer disease, this may involve fibrosis, metaplasia, and/or destructive tissue damage. In obesity, the pathophysiologic consequence of chronic adipose tissue inflammation is a shift in the homeostatic set point, resulting in insulin resistance and disordered glucose homeostasis.

In conclusion, both acute and chronic insulin resistance have complex etiologies, and the components of insulin resistance are unlikely to be the same in all individuals and groups. We have tried to assemble the current knowledge in these wide-ranging areas and have considered these inputs from an integrative physiologic perspective. Clearly there are multiple mechanisms that can have an impact on insulin action, both in the initial stages of positive energy balance (i.e., before weight gain) and during obesity. These center around a complex interplay among nutrient availability, fatty acid metabolism, adipose tissue hypertrophy, and inflammatory pathways. Furthermore, as mentioned at the outset of this Perspective, there are other influences on insulin sensitivity, such as genetics and CNS effects, that have not been discussed in this review. This reinforces the concept that systemic in vivo insulin resistance is not a molecular diagnosis but rather a final general manifestation reflecting the interaction of multiple organ systems and a variety of overlapping but mechanistically distinct signaling and metabolic pathways. While the importance of insulin resistance has been recognized for many years, much work still remains in order to unravel all of its intricacies. 


\section{Acknowledgments}

We thank Jane Kim for thoughtful discussion and assistance with Figure 2. Studies from this laboratory were funded in part by NIH grants DK 033651 and DK 074868. This research was also supported by the Eunice Kennedy Shriver National Institute of Child Health And Human Development (NICHD)/NIH through cooperative agreement U54 HD 012303 as part of the Specialized Cooperative Centers Program in Reproduction and Infertility Research (to J.M. Olefsky); and the University of California Discovery Program Project no. bio03-10383 (BioSTAR) with matching funds from Pfizer Inc. S. Schenk is supported by a Mentor-Based
Postdoctoral Fellowship from the American Diabetes Association awarded to J.M. Olefsky. M. Saberi is supported through an NIH training grant (5 T32DK007494-24), awarded to J.M. Olefsky.

Address correspondence to: Jerrold M. Olefsky, University of California, San Diego, Department of Medicine, 225 Stein Clinical Research Building, 9500 Gilman Dr., La Jolla, California 92093, USA. Phone: (858) 534-6651; Fax: (858) 534-6653; E-mail: jolefsky@ucsd.edu.

Simon Schenk and Maziyar Saberi contributed equally to this work.
1. Ogden, C.L., et al. 2006. Prevalence of overweight and obesity in the United States, 1999-2004. JAMA. 295:1549-1555.

2. Mokdad, A.H., et al. 2003. Prevalence of obesity, diabetes, and obesity-related health risk factors, 2001. JAMA. 289:76-79.

3. Reaven, G.M. 2005. The insulin resistance syndrome: definition and dietary approaches to treatment. Annu. Rev. Nutr. 25:391-406.

4. Facchini, F.S., Hua, N., Abbasi, F., and Reaven, G.M. 2001. Insulin resistance as a predictor of age-related diseases. J. Clin. Endocrinol. Metab. 86:3574-3578.

5. Stumvoll, M., Goldstein, B.J., and van Haeften, T.W. 2005. Type 2 diabetes: principles of pathogenesis and therapy. Lancet. 365:1333-1346.

6. Morino, K., Petersen, K.F., and Shulman, G.I. 2006. Molecular mechanisms of insulin resistance in humans and their potential links with mitochondrial dysfunction. Diabetes. 55(Suppl. 2):S9-S15.

7. Zierath, J.R., and Wallberg-Henriksson, H. 2002. From receptor to effector: insulin signal transduction in skeletal muscle from type II diabetic patients. Ann. N. Y. Acad. Sci. 967:120-134.

8. Hamilton, M.T., Hamilton, D.G., and Zderic, T.W. 2007. The role of low energy expenditure and sitting on obesity, metabolic syndrome, Type 2 diabetes, and cardiovascular disease. Diabetes. 56:2655-2667.

9. Loos, R.J., and Bouchard, C. 2003. Obesity - is it a genetic disorder? J. Intern. Med. 254:401-425.

10. Obici, S., and Rossetti, L. 2003. Minireview: nutrient sensing and the regulation of insulin action and energy balance. Endocrinology. 144:5172-5178.

11. Schwartz, M.W., and Porte, D., Jr. 2005. Diabetes, obesity, and the brain. Science. 307:375-379.

12. Taniguchi, C.M., Emanuelli, B., and Kahn, C.R. 2006. Critical nodes in signalling pathways: insights into insulin action. Nat. Rev. Mol. Cell Biol. 7:85-96.

13. Thirone, A.C., Huang, C., and Klip, A. 2006. Tissue-specific roles of IRS proteins in insulin signaling and glucose transport. Trends Endocrinol. Metab. 17:72-78.

14. Duncan, R.E., Ahmadian, M., Jaworski, K., SarkadiNagy, E., and Sul, H.S. 2007. Regulation of lipolysis in adipocytes. Annu. Rev. Nutr. 27:79-101.

15. Wahren, J., and Ekberg, K. 2007. Splanchnic regulation of glucose production. Annu. Rev. Nutr. 27:329-345

16. Gual, P., Le Marchand-Brustel, Y., and Tanti, J.F. 2005. Positive and negative regulation of insulin signaling through IRS-1 phosphorylation. Biochimie. 87:99-109.

17. Hotamisligil, G.S., et al. 1996. IRS-1-mediated inhibition of insulin receptor tyrosine kinase activity in TNF-alpha- and obesity-induced insulin resistance. Science. 271:665-668.

18. Hirosumi, J., et al. 2002. A central role for JNK in obesity and insulin resistance. Nature. 420:333-336.

19. Aguirre, V., et al. 2002. Phosphorylation of Ser307 in insulin receptor substrate-1 blocks interactions with the insulin receptor and inhibits insulin action. J. Biol. Chem. 277:1531-1537.

20. Karin, M., et al. 2001. Oxidative stress and gene expression: the AP-1 and NF-kappaB connections. Biofactors. 15:87-89.

21. Bandyopadhyay, G.K., Yu, J.G., Ofrecio, J., and Olefsky, J.M. 2005. Increased p85/55/50 expression and decreased phosphotidylinositol 3-kinase activity in insulin-resistant human skeletal muscle. Diabetes. 54:2351-2359.

22. Yuan, M., et al. 2001. Reversal of obesity- and dietinduced insulin resistance with salicylates or targeted disruption of Ikkbeta. Science. 293:1673-1677.

23. Itani, S.I., Ruderman, N.B., Schmieder, F., and Boden, G. 2002. Lipid-induced insulin resistance in human muscle is associated with changes in diacylglycerol, protein kinase C, and IkappaB-alpha. Diabetes. 51:2005-2011.

24. Arkan, M.C., et al. 2005. IKK-beta links inflammation to obesity-induced insulin resistance. Nat. Med. 11:191-198.

25. Cai, D., et al. 2005. Local and systemic insulin resistance resulting from hepatic activation of IKK-beta and NF-kappaB. Nat. Med. 11:183-190.

26. Nguyen, M.T., et al. 2005. JNK and tumor necrosis factor-alpha mediate free fatty acid-induced insulin resistance in 3T3-L1 adipocytes. J. Biol. Chem. 280:35361-35371.

27. Hundal, R.S., et al. 2002. Mechanism by which high-dose aspirin improves glucose metabolism in type 2 diabetes. J. Clin. Invest. 109:1321-1326.

28. Wang, J., et al. 2001. Overfeeding rapidly induces leptin and insulin resistance. Diabetes. 50:2786-2791.

29. Kelley, D.E., et al. 1993. Relative effects of calorie restriction and weight loss in noninsulin-dependent diabetes mellitus. J. Clin. Endocrinol. Metab. 77:1287-1293.

30. Cartee, G.D., and Dean, D.J. 1994. Glucose transport with brief dietary restriction: heterogenous responses in muscles. Am. J. Physiol. 266:E946-E952.

31. Assali, A.R., et al. 2001. Insulin resistance in obesity: body-weight or energy balance? J. Endocrinol. 171:293-298.

32. Gumbs, A.A., Modlin, I.M., and Ballantyne, G.H. 2005. Changes in insulin resistance following bariatric surgery: role of caloric restriction and weight loss. Obes. Surg. 15:462-473.

33. McCurdy, C.E., Davidson, R.T., and Cartee, G.D. 2005. Calorie restriction increases the ratio of phosphatidylinositol 3-kinase catalytic to regulatory subunits in rat skeletal muscle. Am. J. Physiol. Endocrinol. Metab. 288:E996-E1001.

34. Cornier, M.A., Bessesen, D.H., Gurevich, I., Leitner, J.W., and Draznin, B. 2006. Nutritional upregulation of p85alpha expression is an early molecular manifestation of insulin resistance. Diabetologia. 49:748-754.

35. Sun, C., Zhang, F., Ge, X., Yan, T., Chen, X., Shi, X., and Zhai, Q. 2007. SIRT1 improves insulin sensitivity under insulin-resistant conditions by repressing PTP1B. Cell Metab. 6:307-319.

36. Civitarese, A.E., et al. 2007. Calorie restriction increases muscle mitochondrial biogenesis in healthy humans. PLoS Med. 4:e76.

37. Um, S.H., et al. 2004. Absence of S6K1 protects against age- and diet-induced obesity while enhanc- ing insulin sensitivity. Nature. 431:200-205.

38. Tzatsos, A., and Kandror, K.V. 2006. Nutrients suppress phosphatidylinositol 3-kinase/Akt signaling via raptor-dependent mTOR-mediated insulin receptor substrate 1 phosphorylation. Mol. Cell. Biol. 26:63-76.

39. Krebs, M., et al. 2007. The Mammalian target of rapamycin pathway regulates nutrient-sensitive glucose uptake in man. Diabetes. 56:1600-1607.

40. Mauvais-Jarvis, F., et al. 2002. Reduced expression of the murine p85alpha subunit of phosphoinositide 3-kinase improves insulin signaling and ameliorates diabetes. J. Clin. Invest. 109:141-149.

41. Chen, D., et al. 2004. p50alpha/p55alpha phosphoinositide 3-kinase knockout mice exhibit enhanced insulin sensitivity. Mol. Cell. Biol. 24:320-329.

42. Geering, B., Cutillas, P.R., Nock, G., Gharbi, S.I., and Vanhaesebroeck, B. 2007. Class IA phosphoinositide 3-kinases are obligate p85-p110 heterodimers. Proc. Natl. Acad. Sci. U. S. A. 104:7809-7814.

43. Milne, J.C., et al. 2007. Small molecule activators of SIRT1 as therapeutics for the treatment of type 2 diabetes. Nature. 450:712-716.

44. Bordone, L., et al. 2007. SIRT1 transgenic mice show phenotypes resembling calorie restriction. Aging Cell. 6:759-767.

45. Horowitz, J.F., and Klein, S. 2000. Whole body and abdominal lipolytic sensitivity to epinephrine is suppressed in upper body obese women. Am.J. Physiol. Endocrinol. Metab. 278:E1144-E1152.

46. Bachmann, O.P., et al. 2001. Effects of intravenous and dietary lipid challenge on intramyocellular lipid content and the relation with insulin sensitivity in humans. Diabetes. 50:2579-2584.

47. Schenk, S., and Horowitz, J.F. 2007. Acute exercise increases triglyceride synthesis in skeletal muscle and prevents fatty acid-induced insulin resistance. J. Clin. Invest. 117:1690-1698.

48. Santomauro, A.T., et al. 1999. Overnight lowering of free fatty acids with Acipimox improves insulin resistance and glucose tolerance in obese diabetic and nondiabetic subjects. Diabetes. 48:1836-1841.

49. Bajaj, M., et al. 2005. Effect of a sustained reduction in plasma free fatty acid concentration on intramuscular long-chain fatty Acyl-CoAs and insulin action in type 2 diabetic patients. Diabetes. 54:3148-3153.

50. Klein, S., Luu, K., Gasic, S., and Green, A. 1996. Effect of weight loss on whole body and cellular lipid metabolism in severely obese humans. Am.J. Physiol. 270:E739-E745.

51. Coleman, R.A., and Lee, D.P. 2004. Enzymes of triacylglycerol synthesis and their regulation. Prog. Lipid Res. 43:134-176.

52. Summers, S.A. 2006. Ceramides in insulin resistance and lipotoxicity. Prog. Lipid Res. 45:42-72.

53. Liu, L., et al. 2007. Upregulation of myocellular DGAT1 augments triglyceride synthesis in skeletal muscle and protects against fat-induced insulin resistance. J. Clin. Invest. 117:1679-1689.

54. Pan, D.A., et al. 1997. Skeletal muscle triglyceride levels are inversely related to insulin action. Diabe- 
tes. 46:983-988.

55. Goodpaster, B.H., He, J., Watkins, S., and Kelley, D.E. 2001. Skeletal muscle lipid content and insulin resistance: evidence for a paradox in endurance-trained athletes. J. Clin. Endocrinol. Metab. 86:5755-5761.

56. Bandyopadhyay, G.K., Yu, J.G., Ofrecio, J., and Olefsky, J.M. 2006. Increased malonyl-CoA levels in muscle from obese and type 2 diabetic subjects lead to decreased fatty acid oxidation and increased lipogenesis; thiazolidinedione treatment reverses these defects. Diabetes. 55:2277-2285.

57. Holland, W.L., et al. 2007. Inhibition of ceramide synthesis ameliorates glucocorticoid-, saturatedfat-, and obesity-induced insulin resistance. Cell Metab. 5:167-179.

58. Yu, C., et al. 2002. Mechanism by which fatty acids inhibit insulin activation of insulin receptor substrate-1 (IRS-1)-associated phosphatidylinositol 3-kinase activity in muscle. J. Biol. Chem. 277:50230-50236.

59. Sathyanarayana, P., et al. 2002. Activation of the Drosophila MLK by ceramide reveals TNF-alpha and ceramide as agonists of mammalian MLK3. Mol. Cell. 10:1527-1533.

60. Wang, X., Devaiah, S.P., Zhang, W., and Welti, R. 2006. Signaling functions of phosphatidic acid. Prog. Lipid Res. 45:250-278.

61. Jean-Baptiste, G., Yang, Z., Khoury, C., and Greenwood, M.T. 2005. Lysophosphatidic acid mediates pleiotropic responses in skeletal muscle cells. Biochem. Biophys. Res. Commun. 335:1155-1162.

62. Sampson, S.R., and Cooper, D.R. 2006. Specific protein kinase $C$ isoforms as transducers and modulators of insulin signaling. Mol. Genet. Metab. 89:32-47.

63. Stratford, S., DeWald, D.B., and Summers, S.A. 2001. Ceramide dissociates 3 '-phosphoinositide production from pleckstrin homology domain translocation. Biochem. J. 354:359-368.

64. Park, E., Wong, V., Guan, X., Oprescu, A.I., and Giacca, A. 2007. Salicylate prevents hepatic insulin resistance caused by short-term elevation of free fatty acids in vivo. J. Endocrinol. 195:323-331.

65. Kim, J.K., et al. 2004. PKC-theta knockout mice are protected from fat-induced insulin resistance. J. Clin. Invest. 114:823-827.

66. Kim, J.Y., et al. 2007. Obesity-associated improvements in metabolic profile through expansion of adipose tissue. J. Clin. Invest. 117:2621-2637.

67. Monetti, M., et al. 2007. Dissociation of hepatic steatosis and insulin resistance in mice overexpressing DGAT in the liver. Cell Metab. 6:69-78.

68. Houmard, J.A. 2008. Intramuscular lipid oxidation and obesity. Am. J. Physiol. Regul. Integr. Comp. Physiol. 294: R1111-R1116.

69. Mootha, V.K., et al. 2003. PGC-1alpha-responsive genes involved in oxidative phosphorylation are coordinately downregulated in human diabetes. Nat. Genet. 34:267-273.

70. Patti, M.E., et al. 2003. Coordinated reduction of genes of oxidative metabolism in humans with insulin resistance and diabetes: Potential role of PGC1 and NRF1. Proc. Natl. Acad. Sci. U. S. A. 100:8466-8471.

71. Perdomo, G., et al. 2004. Increased beta-oxidation in muscle cells enhances insulin-stimulated glucose metabolism and protects against fatty acid-induced insulin resistance despite intramyocellular lipid accumulation. J. Biol. Chem. 279:27177-27186.

72. Matzinger, O., Schneiter, P., and Tappy, L. 2002. Effects of fatty acids on exercise plus insulin-induced glucose utilization in trained and sedentary subjects. Am. J. Physiol. Endocrinol. Metab. 282:E125-E131.

73. Bonen, A., et al. 2004. Triacylglycerol accumulation in human obesity and type 2 diabetes is associated with increased rates of skeletal muscle fatty acid transport and increased sarcolemmal FAT/CD36. FASEB J. 18:1144-1146.
74. Steinberg, G.R., et al. 2004. AMP-activated protein kinase is not down-regulated in human skeletal muscle of obese females. J. Clin. Endocrinol. Metab. 89:4575-4580

75. Boushel, R., et al. 2007. Patients with type 2 diabetes have normal mitochondrial function in skeletal muscle. Diabetologia. 50:790-796.

76. Nair, K.S., et al. 2008. Asian Indians have enhanced skeletal muscle mitochondrial capacity to produce ATP in association with severe insulin resistance. Diabetes. 57:1166-1175.

77. Handschin, C., et al. 2007. Abnormal glucose homeostasis in skeletal muscle-specific PGC-1alpha knockout mice reveals skeletal muscle-pancreatic beta cell crosstalk. J. Clin. Invest. 117:3463-3474.

78. Pospisilik, J.A., et al. 2007. Targeted deletion of AIF decreases mitochondrial oxidative phosphorylation and protects from obesity and diabetes. Cell. 131:476-491.

79. Bonnard, C., et al. 2008. Mitochondrial dysfunction results from oxidative stress in the skeletal muscle of diet-induced insulin-resistant mice. J. Clin. Invest. 118:789-800.

80. Koves, T.R., et al. 2008. Mitochondrial overload and incomplete Fatty Acid oxidation contribute to skeletal muscle insulin resistance. Cell Metab. 7:45-56.

81. Dube, J.J., et al. 2008. Exercise-induced alterations in intramyocellular lipids and insulin resistance: the athlete's paradox revisited. Am. J. Physiol. Endocrinol. Metab. 294:E882-E888.

82. Izumiya, Y., et al. 2008. Fast/Glycolytic muscle fiber growth reduces fat mass and improves metabolic parameters in obese mice. Cell Metab. 7:159-172.

83. de Luca, C., and Olefsky, J.M. 2008. Inflammation and insulin resistance. FEBS Lett. 582:97-105.

84. Wang, P., Mariman, E., Renes, J., and Keijer, J. 2008. The secretory function of adipocytes in the physiology of white adipose tissue. J. Cell. Physiol. 216:3-13.

85. Shoelson, S.E., Herrero, L., and Naaz, A. 2007. Obesity, inflammation, and insulin resistance. Gastroenterology. 132:2169-2180.

86. Hotamisligil, G.S., Shargill, N.S., and Spiegelman, B.M. 1993. Adipose expression of tumor necrosis factor-alpha: direct role in obesity-linked insulin resistance. Science. 259:87-91.

87. Solinas, G., et al. 2007. JNK1 in hematopoietically derived cells contributes to diet-induced inflammation and insulin resistance without affecting obesity. Cell Metab. 6:386-397.

88. Hosogai, N., et al. 2007. Adipose tissue hypoxia in obesity and its impact on adipocytokine dysregulation. Diabetes. 56:901-911.

89. Wang, B., Wood, I.S., and Trayhurn, P. 2007. Dysregulation of the expression and secretion of inflammation-related adipokines by hypoxia in human adipocytes. Pflugers Arch. 455:479-492.

90. Ye, J., Gao, Z., Yin, J., and He, Q. 2007. Hypoxia is a potential risk factor for chronic inflammation and adiponectin reduction in adipose tissue of ob/ob and dietary obese mice. Am. J. Physiol. Endocrinol. Metab. 293:E1118-E1128.

91. Cinti, S., et al. 2005. Adipocyte death defines macrophage localization and function in adipose tissue of obese mice and humans. J. Lipid Res. 46:2347-2355.

92. Strissel, K.J., et al. 2007. Adipocyte death, adipose tissue remodeling, and obesity complications. Diabetes. 56:2910-2918.

93. Rodriguez, A., Catalan, V., Gomez-Ambrosi, J., and Fruhbeck, G. 2007. Visceral and subcutaneous adiposity: are both potential therapeutic targets for tackling the metabolic syndrome? Curr. Pharm. Des. 13:2169-2175

94. Gregor, M.F., and Hotamisligil, G.S. 2007. Thematic review series: Adipocyte Biology. Adipocyte stress: the endoplasmic reticulum and metabolic disease. J. Lipid Res. 48:1905-1914.

95. Ron, D., and Walter, P. 2007. Signal integration in the endoplasmic reticulum unfolded protein response. Nat. Rev. Mol. Cell Biol. 8:519-529.

96. Eizirik, D.L., Cardozo, A.K., and Cnop, M. 2008. The role for endoplasmic reticulum stress in diabetes mellitus. Endocr. Rev. 29:42-61.

97. Ozcan, U., et al. 2004. Endoplasmic reticulum stress links obesity, insulin action, and type 2 diabetes. Science. 306:457-461.

98. Li, Y., et al. 2005. Free cholesterol-loaded macrophages are an abundant source of tumor necrosis factoralpha and interleukin-6: model of NF-kappaB- and map kinase-dependent inflammation in advanced atherosclerosis. J. Biol. Chem. 280:21763-21772.

99. Ozcan, U., et al. 2006. Chemical chaperones reduce ER stress and restore glucose homeostasis in a mouse model of type 2 diabetes. Science. 313:1137-1140.

100.Yoshiuchi, K., et al. 2008. Direct monitoring of in vivo ER stress during the development of insulin resistance with ER stress-activated indicator transgenic mice. Biochem. Biophys. Res. Commun. 366:545-550.

101.Weisberg, S.P., et al. 2003. Obesity is associated with macrophage accumulation in adipose tissue. J. Clin. Invest. 112:1796-1808.

102. Xu, H., et al. 2003. Chronic inflammation in fat plays a crucial role in the development of obesity-related insulin resistance. J. Clin. Invest. 112:1821-1830.

103.Lumeng, C.N., Bodzin, J.L., and Saltiel, A.R. 2007. Obesity induces a phenotypic switch in adipose tissue macrophage polarization. J. Clin. Invest. 117:175-184.

104.Lumeng, C.N., Deyoung, S.M., Bodzin, J.L., and Saltiel, A.R. 2007. Increased inflammatory properties of adipose tissue macrophages recruited during diet-induced obesity. Diabetes. 56:16-23.

105. Nguyen, M.T., et al. 2007. A subpopulation of macrophages infiltrates hypertrophic adipose tissue and is activated by FFAS via TLR2, TLR4 and JNK-dependent pathways. J. Biol. Chem. 282:35279-35292.

106. Hevener, A.L., et al. 2007. Macrophage PPAR gamma is required for normal skeletal muscle and hepatic insulin sensitivity and full antidiabetic effects of thiazolidinediones. J. Clin. Invest. 117:1658-1669.

107. Greten, F.R., et al. 2004. IKKbeta links inflammation and tumorigenesis in a mouse model of colitis-associated cancer. Cell. 118:285-296.

108. De Taeye, B.M., et al. 2007. Macrophage TNF-alpha contributes to insulin resistance and hepatic steatosis in diet-induced obesity. Am. J. Physiol. Endocrinol. Metab. 293:E713-E725.

109. Hirasaka, K., et al. 2007. Deficiency of Cbl-b gene enhances infiltration and activation of macrophages in adipose tissue and causes peripheral insulin resistance in mice. Diabetes. 56:2511-2522.

110. Mantovani, A., et al. 2004. The chemokine system in diverse forms of macrophage activation and polarization. Trends Immunol. 25:677-686.

111.Lebre, M.C., et al. 2005. Differential expression of inflammatory chemokines by Th1- and Th2cell promoting dendritic cells: a role for different mature dendritic cell populations in attracting appropriate effector cells to peripheral sites of inflammation. Immunol. Cell Biol. 83:525-535.

112. Bouhlel, M.A., et al. 2007. PPARgamma activation primes human monocytes into alternative M2 macrophages with anti-inflammatory properties. Cell Metab. 6:137-143.

113. Odegaard, J.I., et al. 2007. Macrophage-specific PPARgamma controls alternative activation and improves insulin resistance. Nature. 447:1116-1120.

114.Lee, J.Y., Sohn, K.H., Rhee, S.H., and Hwang, D. 2001. Saturated fatty acids, but not unsaturated fatty acids, induce the expression of cyclooxygenase2 mediated through Toll-like receptor 4. J. Biol. Chem. 276:16683-16689.

115.Lee, J.Y., et al. 2003. Reciprocal modulation of Toll-like receptor-4 signaling pathways involving MyD88 and phosphatidylinositol 3-kinase/AKT by 
saturated and polyunsaturated fatty acids. J. Biol. Chem. 278:37041-37051.

116.Shi, H., et al. 2006. TLR4 links innate immunity and fatty acid-induced insulin resistance. J. Clin. Invest. 116:3015-3025.

117.Tsukumo, D.M., et al. 2007. Loss-of-function mutation in Toll-like receptor 4 prevents dietinduced obesity and insulin resistance. Diabetes. 56:1986-1998.

118.Cani, P.D., et al. 2007. Metabolic endotoxemia initiates obesity and insulin resistance. Diabetes. 56:1761-1772.

119. Chen, A., et al. 2005. Diet induction of monocyte chemoattractant protein-1 and its impact on obesity. Obes. Res. 13:1311-1320.

120. Weisberg, S.P., et al. 2006. CCR2 modulates inflammatory and metabolic effects of high-fat feeding. J. Clin. Invest. 116:115-124.

121. Kanda, H., et al. 2006. MCP-1 contributes to macrophage infiltration into adipose tissue, insulin resistance, and hepatic steatosis in obesity. J. Clin. Invest. 116:1494-1505.

122.Inouye, K.E., et al. 2007. Absence of CC chemokine ligand 2 does not limit obesity-associated infiltration of macrophages into adipose tissue. Diabetes. 56:2242-2250.
123. Kirk, E.A., Sagawa, Z.K., McDonald, T.O., O’Brien, K.D., and Heinecke, J.W. 2008. Macrophage chemoattractant protein-1 deficiency fails to restrain macrophage infiltration into adipose tissue. Diabetes. 57:1254-1261.

124. Nomiyama, T., et al. 2007. Osteopontin mediates obesity-induced adipose tissue macrophage infiltration and insulin resistance in mice. J. Clin. Invest. 117:2877-2888.

125.Goodpaster, B.H., Thaete, F.L., and Kelley, D.E. 2000. Thigh adipose tissue distribution is associated with insulin resistance in obesity and in type 2 diabetes mellitus. Am. J. Clin. Nutr. 71:885-892. 